Chapman University

Chapman University Digital Commons

Physical Therapy Faculty Articles and Research

Physical Therapy

2012

\title{
Investigation into the Functional Mobility Difference between Obese and Non-Obese Elderly
}

Xuefang Wu

Virginia Tech

Han T. Yeoh

Virginia Tech

Rahul Soangra

ChapmanUniversity, soangra@chapman.edu

Thurmon Lockhart

Virginia Tech

Follow this and additional works at: https://digitalcommons.chapman.edu/pt_articles

Part of the Movement and Mind-Body Therapies Commons, Musculoskeletal System Commons, and the Physical Therapy Commons

\section{Recommended Citation}

Wu X, Yeoh HT, Soangra R, Lockhart TE. Investigation into the functional mobility difference between obese and non-obese elderly. Proc Hum Factors Ergon Soc Annu Meet. 2012;56:1814-1816. doi: 10.1177/1071181312561365

This Conference Proceeding is brought to you for free and open access by the Physical Therapy at Chapman University Digital Commons. It has been accepted for inclusion in Physical Therapy Faculty Articles and Research by an authorized administrator of Chapman University Digital Commons. For more information, please contact laughtin@chapman.edu. 


\section{Investigation into the Functional Mobility Difference between Obese and Non-Obese Elderly}

\section{Comments}

This article was originally published in Proceedings of the Human Factors and Ergonomics Society Annual Meeting, volume 56, in 2012. DOI: 10.1177/1071181312561365

\section{Copyright}

Not subject to U.S. copyright restrictions. 


\title{
Investigation into the Functional Mobility Difference between Obese and Non-Obese Elderly
}

\author{
Xuefang $\mathrm{Wu}^{1}$, Han T Yeoh ${ }^{1}$, Rahul Soangra ${ }^{2}$, Thurmon E Lockhart ${ }^{1,2}$ \\ 1. Locomotion Research Laboratory, Grado Department of Industrial and System Engineering, \\ Virginia Tech, Blacksburg, VA, 24060 \\ 2. Department of Biomedical Engineering, Virginia Tech, Blacksburg, VA, 24060
}

\begin{abstract}
Obese aging population is increasing in the United States, and obese elderly experience fall twice as frequent as their lean counterparts. However, the mechanisms of older obese adults fall are still not clear. It is not known whether the obese elderly has more functional mobility impairments than their lean counterparts, and consequently have increased risks of falls. It was hypothesized that obese elderly have more functional mobility impairments compared with their healthy weight counterparts. Six lean and six obese community-dwelling elderly participated in the study. "Timed up \& go" test was used to quantify the functional mobility for both lean and obese elderly. Stopwatch and custom-made inertial measurement units were used to obtain the temporal and kinematic parameters. The results showed that there is no significant difference in overall time to complete the "timed up \& go" task, but significant difference in anterior posterior acceleration, time to reach the peak extension angular velocity from initiation and double support time between lean and obese groups of participants. Therefore, we concluded that older obese adults have some functional mobility impairments compared with their lean counterparts but the completion time of the "timed up \& go" test may not be able to differentiate these individuals. Our results also suggested that obese elderly might have more muscular impairments than their healthy weight counterparts, which can result in higher fall risks. Future studies are warranted to investigate the mechanisms of increased fall risks among obese elderly.
\end{abstract}

Introduction

Falls are the most common cause of injuries among older adults aged 65 and above (CDC, 2008). Numerous studies have investigated the mechanisms of increased fall risks associated with elderly. The etiology of falls amongst senior citizens is generally considered multi-factorial (American Geriatrics Society, 2001; Perell et al., 2001; Tinnetti et al., 1988). The most likely intrinsic risk factors that cause falls are: advanced age, chronic diseases, muscular weakness or reduced muscle mass, a previous history of falls, and deficits in gait and balance (Perell et al., 2001; Tinnetti et al., 1988). Among all elderly, obese elderly fell almost twice as frequently as their lean counterparts every year (Wu et al., 2012). However, to our knowledge, no study to date investigated mechanisms of falls among obese elderly. As the number of obese elderly is increasing in the United States (Mokdad et al., 2001), it is important to reveal the mechanisms of more falls among these individuals in order to help improve the fall prevention and screening tests for the aging population. Therefore, the aim of this study was try to reveal the mechanisms of increased fall risks associated with obese elderly.

Studies indicated that obese elderly walked with lower speed compared with their lean counterparts (Seung-uk et al., 2010), which may indicate their impaired mobility (Houston et al., 2009) that can lead to increased fall risks. However, no study to date directly compared the mobility impairments of obese elderly with their lean counterparts. Literatures suggested that functional mobility test such as "timed up \& go" test, which is objective, valid and reliable (Podsiadlo and Richardson, 2001; Shumway-Cook et al., 2000) can be used to identify fall risks among elderly (Greene et al., 2010), because it evaluates four factors that influence the fall risks: 1) strength in lower extremities 2) coordination 3) balance 4) gait (Gine-Garriga et al., 2009). Therefore, the objective of this study was to investigate whether obese elderly have more functional mobility impairments than their healthy weight counterparts, using "timed up \& go" test. It was hypothesized that obese elderly have more functional mobility impairments compared with their healthy weight counterparts.

Method

Participants

Six non-obese $(\mathrm{BMI}<30)$ (1male and 5 females) and six obese (BMI $>30)$ (1 male and 5 females) community-dwelling elderly (aged 65-84) participated in this study. All participants were able to rise from the chair without any assistance. Participants with walking aid were excluded from this study. Participants' anthropometric data were presented in Table 1.

Table 1. Anthropometric data for obese/non-obese participants

\begin{tabular}{|l|l|l|l|l|}
\hline Group & Age & Weight $(\mathrm{kg})$ & Height $(\mathrm{m})$ & BMI \\
\hline Non-obese & $76.7 \pm 6.7$ & $67.4 \pm 12.5$ & $1.63 \pm 0.04$ & $25.4 \pm 3.86$ \\
\hline Obese & $75.8 \pm 2.2$ & $99.3 \pm 19.3$ & $1.68 \pm 0.12$ & $34.8 \pm 3.33$ \\
\hline
\end{tabular}

Note: Results are in the format of mean \pm standard deviation. 


\section{Equipment}

Three custom-made wireless inertial measurement units (IMUs) (TEMPO: Technology-Enabled Medical Precision Observation, which was manufactured in collaboration with the inertia team from University of Virginia. The TEMPO system consists of MMA7261QT tri-axial accelerometers and IDG-300 (x and y plane) and ADXRS300A (z plane) uniaxial gyroscopes.) were attached on participants' sternum and both ankles (lateral sides) to obtain the temporal and kinematic data. The data acquisition was carried out using a Bluetooth adapter and laptop via a custom-made LabView VI (Barth et al., 2009). Temporal and kinematic data were acquired with sampling frequency of $128 \mathrm{~Hz}$. Stopwatch was also used to time the trials. A standard armchair (seat height of $43 \mathrm{~cm}$, arm height of $65 \mathrm{~cm}$ ) was used for participants to perform the "timed up \& go" task.

\section{Experiment protocol}

Wearing their own footwear, participants started with sitting posture and their back against the chair, arms resting on the armrest, thighs paralleled with their feet. They were instructed that, on the word "go" they should get up and walk at a comfortable and safe pace to a line on the floor 3 meters away from the chair, turn, and return to the chair and sit down again. The participants had a chance to walk through the test once before being timed in order to become familiar with the test. The two consequent trials were timed and recorded for the experiment.

\section{Data Analysis}

The mean of the two timed trials were used to represent the temporal and kinematic characteristics each participant during the task. Transitional phase time and peak velocity and acceleration during the transitional phase were calculated using MATLAB (the Mathworks, Inc., MA, USA) from the IMU data. Ensemble Empirical Mode Decomposition (EEMD) (Huang et al., 1999)-Golay was used to denoise the IMU data. The chosen number of ensembles was 100 with the ratio of the standard deviation of the added noise to that of the signal as 0.2 . The calculated parameters included anterior posterior peak acceleration (AP acc), difference between the peak flexion angular velocity and peak extension angular velocity (FE AV dif), peak flexion angular velocity (PFAV), peak extension angular velocity (PEAV), initial flexion angular acceleration (IFAA), before seat-off flexion angular deceleration (BS F Adec), after seat-off extension angular acceleration (AS E Aacc), trunk forward jerk (TFJ), time from start to peak flexion angular velocity (T1), time from start to seat-off (T2), time from start to peak extension angular velocity (T3), time from start to the first toe off (T4), time from start to first heel contact (T5), time from start to second toe off (T6), time from start to the second heel contact (T7), time for the first gait cycle (T8), time for single stance phase in the gait cycle (T9), 1/2 double support time (T10), time in between peak flexion and extension momentums (T11), time in between peak flexion angular velocity and seat off (T12), time in between seat off and peak extension angular velocity (T13) and time of "timed up and go" task completion (14). One-way between-subject ANOVA was performed to determine the statistical difference using JMP, with significance level of $<0.05$.

Results

The mean and standard deviation with the statistical results were provided in Table 2. The one-way ANOVA indicated that the obese participants stood up with less anterior posterior acceleration $(\mathrm{F}(1,10)=5.0086, \mathrm{p}=0.0492)$, but similar peak flexion and extension angular velocity, similar initial flexion angular acceleration, before seat-off flexion angular acceleration, after seat-off extension angular acceleration, trunk forward jerk. All participants spent relatively similar time in reaching the point of peak flexion angular velocity, peak flexion angular velocity, first toe off, first heel contact, second toe off, second heel contact from the initial movements, but obese participants spent significant longer time from initial movement to peak extension angular velocity $(\mathrm{F}(1,10)=5.7590, \mathrm{p}=0.0373)$. Additionally, obese and nonobese participants spent similar time in single support, but obese subjects spent significant longer time in double support $(\mathrm{F}(1,10)=4.9624, \mathrm{p}=0.05)$. Furthermore, it took similar time for both group of participants from peak flexion momentums to peak extension momentums, from peak flexion angular velocity to seat off, from seat off to peak extension and similar time in completion the "time up \& go" test.

Table 2. Results

\begin{tabular}{|c|c|c|c|}
\hline Parameter & Non-obese & Obese & ANOVA \\
\hline AP acc (m/s $\left.{ }^{2}\right)$ & $0.27 \pm 0.12$ & $0.08 \pm 0.09$ & $*$ \\
\hline $\begin{array}{c}\text { FE AV dif } \\
(d e g / s)\end{array}$ & $229.24 \pm 93.46$ & $282.46 \pm 140.38$ & N.S. \\
\hline PFAV (deg/s) & $99.64 \pm 59.16$ & $120.58 \pm 75.39$ & N.S. \\
\hline PEAV (deg/s) & $129.60 \pm 50.30$ & $161.89 \pm 95.43$ & N.S. \\
\hline IFAA (deg/s $\left.{ }^{2}\right)$ & $2.09 \pm 0.94$ & $3.39 \pm 5.69$ & N.S. \\
\hline $\begin{array}{c}\text { BS F Adec } \\
\left(\text { deg/s }{ }^{2}\right)\end{array}$ & $-4.77 \pm 3.32$ & $-6.18 \pm 6.21$ & N.S. \\
\hline $\begin{array}{c}\text { AS E Aacc } \\
\left(\text { deg/s }{ }^{2}\right)\end{array}$ & $-5.41 \pm 4.59$ & $-6.63 \pm 5.03$ & N.S. \\
\hline TFJ (m/s $)$ & $-0.02 \pm 0.01$ & $-0.02 \pm 0.01$ & N.S. \\
\hline T1(s) & $1.28 \pm 1.12$ & $0.84 \pm 0.35$ & N.S. \\
\hline T2 (s) & $0.82 \pm 0.54$ & $0.65 \pm 0.30$ & N.S. \\
\hline T3 (s) & $0.47 \pm 0.09$ & $0.49 \pm 0.24$ & $*$ \\
\hline T4 (s) & $1.44 \pm 1.13$ & $0.92 \pm 0.56$ & N.S. \\
\hline T5 (s) & $1.90 \pm 1.13$ & $1.35 \pm 0.59$ & N.S. \\
\hline T6 (s) & $1.95 \pm 1.22$ & $1.43 \pm 0.59$ & N.S. \\
\hline T7 (s) & $1.96 \pm 0.35$ & $1.95 \pm 0.37$ & N.S. \\
\hline T8 (s) & $0.90 \pm 0.04$ & $1.04 \pm 0.18$ & N.S. \\
\hline T9 (s) & $0.90 \pm 0.03$ & $0.97 \pm 0.12$ & N.S. \\
\hline T10 (s) & $0.03 \pm 0.04$ & $0.07 \pm 0.07$ & $*$ \\
\hline T11 (s) & $0.45 \pm 0.19$ & $0.34 \pm 0.15$ & N.S. \\
\hline T12 (s) & $0.27 \pm 0.15$ & $0.22 \pm 0.09$ & N.S. \\
\hline T13 (s) & $0.18 \pm 0.09$ & $0.12 \pm 0.11$ & N.S. \\
\hline T14 (s) & $9.00 \pm 2.27$ & $8.40 \pm 2.46$ & \\
\hline
\end{tabular}

Note: * stands for significant, N.S. stands for not significant. Results are in the format of mean \pm standard deviation. 


\section{Discussion}

This study examined the relationship between obesity and functional mobility that may be used to quantify fall risks among aging individuals. Several distinct events can be identified from the "timed up \& go" test using inertial measurement units (TEMPO). These events were continuous and consistent for all participants. They are initial movements, peak flexion angular velocity, seat-off, peak extension angular velocity, gait initiation, first toe off, first heel contact, second toe off, and second heel contact. These events were in agreements with previous studies using camera and force plates (Buckley et al., 2005; Kerr et al., 2007).

Our results indicated that there was no significant difference in overall time to complete the "timed up \&go" task for both obese and non-obese elderly. The task completion time is strongly correlated to level of functional mobility (the more time taken, the more dependent in activities of daily living) (Podsiadlo and Richardson, 2001). As a result, there was no significant difference in functional mobility between obese and non-obese elderly as quantified by the overall time of "timed up \& go" test. In terms of the peak anterior posterior (AP) acceleration, obese participants performed with significant less AP accelerations. This may be in relation to the excessive body mass, because when similar muscular forces and torques were generated, more body mass will result in less accelerations. Obese elderly also spent significant longer time in reaching the peak extension angular velocity (T3), which can suggest slowed extension momentum generation. Slowed momentum generation may indicate the inability of obese individuals to maintain timing sequence in postural transitions due to the excessive body weight. The reduced capability of transfer the heavier body from flexion to upright can indicate the lower extremity muscular impairments (LaRoche et al., 2011). Furthermore, significant longer double support time (T10) among obese participants found in this study was in consistent with previous study investigated the gait of obese elderly (LaRoche et al., 2011). Due to the excessive body weight, more load must be lifted by the stance hip abductor muscles (Eng et al., 1995) during gait. Increased double-support time may be fine-tuning mechanisms to minimize the time spent in single-support when increased joint effort is required to support increased body mass with only one limb. However, slowed weight acceptance during the double support phase can lead to slowed transfer of the whole body center-of-mass and fall may happen (Lockhart et al., 2003). Further study is warranted to investigate the muscle strength of obese elderly and its relation to the higher fall risks.

\section{Conclusion}

Based on the data we collected from this study, we concluded that older obese adults have some functional mobility impairments compared with their lean counterparts but the completion time of the "timed up \& go" test may not be able to differentiate these individuals. Our results also suggested that obese elderly might have more muscular impairments than their healthy weight counterparts, which can result in higher fall risks. Future studies are warranted to investigate the mechanisms of increased fall risks among obese elderly.

\section{References}

American Geriatrics Society, 2001. Guideline for the prevention of falls in older persons. Journal of American Geriatric Society, 49: 664-672.

Barth, A.T., Hanson, M.A., Powell Jr, H.C., Lach, J. (2009). TEMPO 3.1: A body area sensor network platform for continuous movement assessment. IEEE transaction computer society, 71-76.

Buckley, T. A., Pisikoulis, C., Hass, C.J., 2005. Dynamic postural stability during sit-to-walk in Parkinson disease patients. Movement disorders, 23: $1274-1280$.

Centers For Disease Control and Prevention, 2008. National Center for Injury Prevention and Control, Division of Unintentional Injury Prevention. Falls among older adults: An overview. Available at http://www.cdc.gov/ncipc/ factsheets/adultfalls.htm. Accessed June 25, 2012.

Eng, J.J., Winter, D.A., 1995. Kinetic analysis of the lower limbs during walking. What information can be gained from a three-dimensional model? Journal of biomechanics, 28: 753-758.

Gine-Garriga, M., Guerra, M., Mari-Dell'Olmo, M., Martin, C., Unnithan, V.B., 2009. Sensitivity of a modified version of the 'timed get up and go' test to predict fall risk in the elderly: a pilot study. Archives of Gerontology and Geriatrics, 49(1): 60-66.

Greene, B.R., O’Donovan, A., Romero-Ortuno, R., Cogan, L., Scanaill, C.N., Kenny, R.A., 2010. Qualitative falls risk assessment using the timed up and go test. IEEE Transaction Biomedical Engineering, 57: 2918-2926.

Houston, D.K., Ding, J., Nicklas, B.J., Harris, T.B., Lee, J.S., Nevitt, M.C., Rubin, S.M., Tylavsky, F.A., Kritchevsky, S.B., 2009. Overweight and obesity over the adult life course and incident mobility limitation in older adults: the health, aging and body composition study. American Journal of Epidemiology, 169: 927-936.

Huang, N.E., Shen, Z., Long, S.R., 1999. A new view of nonlinear water waves: the Hilbert spectrum. Annual review of fluid mechanics, 31: 417-457.

Kerr, A., Rafferty, D., Kerr, K.M., Durward, B., 2007. Timing phases of the sit-to-walk movement validity of a clinical test. Gait \& Posture, 26: 11-16.

LaRoche, D.P., Kralian, R.J., Millet, E.D., 2011. Fat mass limits lowerextremity relative strength and maximal walking performance in older women. Journal of electromyography and kinesiology, 21: 754-761.

Lockhart, T.E., Woldstad, J., Smith, J.L., 2003. Effects of age-related gait changes on biomechanics of slips and falls. Ergonomics, 46(12): 11361160 .

Mokdad, A.H., Bowman, B.A., Ford, E.S., Vinicor, F., Marks, J.S., Koplan, J.P., 2001. The continuing epidemics of obesity and diabetes in the United States. Journal of the American Medical Association, 286: 1195-1200.

Perell, K.L., Nelson, A., Goldman, R., Luther, S.L., Prieto-Lewis, N., Rubenstein, L.Z., 2001. Fall risk measures: an analytic review. Journal of Gerontology: Medical Science, 56A: 186-193.

Podsiadlo, D., and Richardson, S., 1991. The timed "up \& go": a test of basic functional mobility for frail elderly persons. Journal of the American Geriatrics society, 39: 142-148.

Seung-uk, K.O., Stenholm, S., Ferrucci, L., 2010. Characteristics gait patterns in older adults with obesity-results from the Baltimore longitudinal study of aging. Journal of Biomechanics, 43: 1104-1110.

Shumway-Cook, A., Brauer, S., \& Woollacott, M., 2000. Predicting the probability for falls in community-dwelling older adults using the timed up \& go test. Physical Therapy, 80(9): 896-903.

Spyroloulos, P., Pisciotta, J.C., Pavlou, K.N., Cairns, M.A., Simon, S.R., 1991. Biomechanical gait analysis in obese men. Archival of Physical Medical Rehabilitation, 72: 1065-1070.

Tinnetti, M.E., Speechley, M., Ginter, S.F., 1988. Risk factors for falls among elderly persons living in the community. New England Journal of Medicine, 319: 1701-1707.

Wearing, S.C., Henning, E.M., Byrne, N.M., Steele, J.R., Hills, A.P., 2006. The biomechanics of restricted movement in adult obesity. The international association for the study of obesity, 7: 13-24.

Wu, X., Lockhart, T.E., Yeoh, H. T., 2012. Effects of obesity on slipinduced fall risks among young male adults. Journal of Biomechanics, 45(6): 1042-1047. 